\title{
Are young Iranian women with metabolically healthy obesity at increased risk of CVD incidence?
}

\author{
Jovens iranianas obesas metabolicamente saudáveis têm maior risco de incidência de DCV? \\ Seyed Ahmad Hosseini,2, Vahideh Aghamohammadi³, Damoon Ashtary-Larky², Meysam Alipour² (D), \\ Matin Ghanavati ${ }^{2}$, Nasrin Lamuchi-Deli ${ }^{4}$
}

\begin{abstract}
Background: The association between the Metabolically Healthy Obese (MHO) phenotype in the absence of metabolic syndrome and subsequent cardiovascular disease remains unclear. Objectives: We examined the association between MHO and CVD risk in young Iranian women. Methods: We studied 183 women aged 20-35 years from a population of 308 candidates. We classified participants into 4 phenotypes. We measured body composition, blood pressure, and biochemical factors in all participants. Results: The Metabolically Healthy Normal Weight (MHNW) and Normal Weight Obese (NWO) phenotypes had no statistical differences in any biochemistry variables. FBS, TG, LDL/HDL, Cholesterol/HDL, hs-CRP, and atherogenic index of plasma (AIP) were all higher in Metabolically Unhealthy Obese $(\mathrm{MUO})$ than $\mathrm{MHO}$ individuals, whereas $\mathrm{HDL}$ was higher in $\mathrm{MHO}$ than in $\mathrm{MUO}$ individuals. LDL/HDL and hs-CRP were higher in MHO participants than MHNW participants, whereas HDL-c was higher in MHNW than MHO. Conclusions: Results of the present study demonstrate that young women displaying the MHO phenotype have a favorable metabolic profile as shown by lower FBS, TG, LDL-c/HDL, Cho/HDL, hs-CRP, and AIP and higher HDL levels than the MUO phenotype. However, MHO individuals were still at greater risk of CVD incidence (lower HDL and higher hs-CRP levels) than MHNW individuals.
\end{abstract}

Keywords: metabolically healthy obese; atherogenic index of plasma; cardiovascular disease.

\begin{abstract}
Resumo
Contexto: A associação entre o fenótipo obeso metabolicamente saudável (OMS) na ausência de síndrome metabólica e doença cardiovascular subsequente permanece incerta. Objetivos: Examinamos a associação entre o fenótipo OMS e risco de DCV em jovens iranianas. Métodos: Analisamos 183 mulheres com idade de 20-35 anos de uma população de 308 candidatas. Classificamos as participantes em quatro fenótipos. Mensuramos composição corporal, pressão arterial e fatores bioquímicos em todas as participantes. Resultados: Os fenótipos com peso normal metabolicamente saudável (PNMS) e obeso com peso normal não apresentaram diferenças estatísticas em nenhuma das variáveis bioquímicas. Os níveis de glicemia sanguínea em jejum (GS)), triglicerídeos (TG), relação LDL/HDL, HDL, proteína $C$ reativa ultrassensível (PCR-us) e índice aterogênico do plasma (IAP) foram mais elevados em obesas metabolicamente não saudáveis (OMNS) do que em indivíduos OMSs, enquanto o HDL foi maior em OMSs do que em indivíduos OMNSs. A relação LDL/HDL e o nível de PCR-us foram mais elevados em participantes OMSs do que em participantes com PNMS, enquanto o HDL foi maior naquelas com PNMS do que nas OMSs. Conclusões: Os resultados do presente estudo demonstram que mulheres jovens com o fenótipo OMS têm um perfil metabólico favorável, conforme demonstrado pelos níveis menores de CSJ, TG, relação LDL/HDL, HDL, PCR-us e IAP e pelos níveis maiores de HDL em comparação às mulheres com o fenótipo OMNS. Entretanto, indivíduos OMSs ainda apresentavam maior risco de DCV incidente (níveis menores de HDL e maiores de PCR-us) do que indivíduos com PNMS.
\end{abstract}

Palavras-chave: obeso metabolicamente saudável; índice aterogênico do plasma; doença cardiovascular.

How to cite: Hosseini SA, Aghamohammadi V, Ashtary-Larky D, Alipour M, Ghanavati M, Lamuchi-Deli N. Are young Iranian women with metabolically healthy obesity at increased risk of CVD incidence? J Vasc Bras. 2020;19:e20190106. https://doi.org/10.1590/1677-5449.190106

\footnotetext{
'Ahvaz Jundishapur University of Medical Sciences, Hyperlipidemia Research Center, Ahvaz, Iran.

${ }^{2}$ Ahvaz Jundishapur University of Medical Sciences, Nutrition and Metabolic Diseases Research Center, Ahvaz, Iran.

${ }^{3}$ Khalkhal University of Medical Sciences, Department of Nutrition, Khalkahl, Iran.

${ }^{4}$ Ahvaz Jundishapur University of Medical Sciences, School of Medicine, Department of Clinical Biochemistry, Ahvaz, Iran.

Financial support: None.

Conflicts of interest: No conflicts of interest declared concerning the publication of this article.

Submitted: August 22, 2019. Accepted: April 20, 2020.
} 


\section{INTRODUCTION}

Obesity is a key risk factor for cardiovascular diseases (CVD), diabetes, hypertension, various types of cancer, mental health, and increased mortality. The obesity rate has tripled over the last two decades in developing countries, including Iran. ${ }^{1,2}$

However, not all obese individuals show an increased risk of mortality. The metabolically healthy obesity (MHO) phenotype has been recognized since the 1980s and encompasses obese individuals who are metabolically healthy despite having an excessive store of body fat and elevated BMI. ${ }^{3}$ Individuals with MHO exhibit a favorable metabolic profile that is determined by a high level of insulin sensitivity, lack of hypertension, and favorable lipid, and inflammatory profiles. ${ }^{3,4}$ The MHO phenotype is the result of various underlying mechanisms and interactions between genetic, behavioral, and environmental agents that have not been elucidated. ${ }^{5}$ The results of previous studies evaluating the effect of MHO on health outcomes remain controversial. ${ }^{6-8}$ There is no consensus on unique criteria that can be used to define $\mathrm{MHO}$ and consequently prevalence rates of the MHO phenotype differ considerably among studies ( 6 to $40 \%$ in the obese population). ${ }^{9}$ The main obstacles to estimating the true prevalence of $\mathrm{MHO}$ are related to the criteria used to define it, the study design, and other factors such as ethnicity, sex, age, and lifestyle..$^{6-8}$ Considering the number of serious health problems associated with obesity, research studying the MHO phenotype may help to identify at-risk obese individuals, support development of better interventions for obese patients, and lead to a novel comprehension of the pathophysiology of obesity.

Since the association between presence of MHO in the absence of metabolic syndrome and subsequent cardiovascular disease remains unclear, we examined the association between $\mathrm{MHO}$ and CVD risk in young Iranian women.

\section{METHODS}

\section{Participants}

The present cross-sectional study was carried out with subjects who were referred to a nutrition clinic, in Ahvaz, Iran. We studied 183 women aged 20-35 years who were selected from a population of 308 candidates. We excluded 125 women who met exclusion criteria including pregnancy, breastfeeding, consumption of any drugs, eating disorders, diabetes, cardiovascular disease, kidney disorders, thyroid disorders, digestive and respiratory diseases, cancer, consumption of more than $300 \mathrm{mg}$ of caffeine daily, and moderate or severe physical activity). Inclusion criteria were regular 28-d menstrual cycles, no physical activity, no smoking, no alcohol consumption, no use of any supplements, and no weight changes in the preceding 6 months. All 183 women signed written informed consent forms. The study protocol was approved by the Ethics Committee at the Ahvaz Jundishapur University of Medical Sciences (IR.AJUMS.REC.1394.489). Participants with metabolic syndrome were diagnosed according to the NCEP ATP III definition, i.e., meeting at least three of the following criteria: waist circumference $\geq 35$ inches (women), blood pressure $\geq 130 / 85 \mathrm{mmHg}$, fasting triglyceride (TG) level $\geq 150 \mathrm{mg} / \mathrm{dL}$, fasting high-density lipoprotein (HDL) cholesterol level less than $50 \mathrm{mg} / \mathrm{dL}$ (for women), and fasting blood sugar $(\mathrm{FBS}) \geq 100 \mathrm{mg} / \mathrm{dL} .{ }^{10}$ We classified participants according to 4 phenotypes based on body mass index (BMI) and metabolic syndrome criteria (Table 1).

\section{Measurements}

A direct segmental multi-frequency bioelectrical impedance method (Inbody 270, Biospace, Korea) was used to calculate body weight and body composition. Waist circumference (WC) was determined in the standing position using a tape with an accuracy of $1.0 \mathrm{~cm}$, above the iliac crest and just below margin of the lowest rib, at the end of the normal expiration. For the hip circumference measurement, the tape was put around the point with the maximum circumference over the buttocks. ${ }^{11}$ The waist-to-hip ratio (WHR) was calculated as waist measurement divided by hip measurement $(\mathrm{W} / \mathrm{H})$. Blood pressure was determined using an automatic blood pressure monitor (BM65, Beurer, Germany) after subjects had been at rest for more than 10 minutes. Measurements were taken in triplicate and the mean was considered for each subject. In this study, RMR was measured by indirect calorimetry (FitMate, Cosmed, Rome, Italy), using resting oxygen uptake ( $\mathrm{VO} 2)$.

For biochemical measurements, $8 \mathrm{cc}$ fasting blood samples were drawn from each subject between days 8 and 12 of their preovulation phase. Blood glucose,

Table 1. Criteria used for diagnosis of metabolically obese phenotypes.

\begin{tabular}{lcc}
\hline \multicolumn{1}{c}{ Inflammatory obese phenotypes } & Obesity status & Metabolic syndrome \\
\hline Metabolically healthy normal weight (MHNW) & $<25\left(\mathrm{~kg} / \mathrm{m}^{2}\right)$ & No \\
Normal weight obese (NWO) & $<25\left(\mathrm{~kg} / \mathrm{m}^{2}\right)$ and body fat $>30 \%$ & Yes/No \\
Metabolically healthy obese (MHO) & $\geq 25\left(\mathrm{~kg} / \mathrm{m}^{2}\right)$ & No \\
Metabolically unhealthy obese (MUO) & $\geq 25\left(\mathrm{~kg} / \mathrm{m}^{2}\right)$ & Yes \\
\hline
\end{tabular}


total cholesterol, LDL-c, HDL-c, and triglycerides were quantified by enzymatic methods with kits from Pars- Azmoon (Tehran, Iran). Insulin concentration and high-sensitivity C-reactive protein (hsCRP) were measured with ELISA kits (Diaplus Inc., Canada). Homeostasis model assessment - insulin resistance (HOMA-IR) was determined using the following formula: fasting glucose $(\mathrm{mg} / \mathrm{dL}) \times$ fasting insulin $(\mu \mathrm{u} / \mathrm{mL}) / 405$.

QUICKI was measured with the following formula $=1 /[\log ($ fasting insulin $(\mu \mathrm{U} \mathrm{mL}))+\log$ (fasting glucose $(\mathrm{mg} / \mathrm{dL}))] 20$ / (fasting C-peptide $\times$ fasting plasma glucose). ${ }^{12}$

\section{Statistical analyses}

All statistical analyses were carried out using IBM SPSS Statistics software version 24 (IBM SPSS Statistics, Armonk, USA). The normality of variables was established using the Kolmogorov- Smirnov test. ANOVA was applied to test differences within groups. Comparisons of variables that were different between groups were performed using the Tukey test for post-hoc analyses. A p value less than 0.05 was considered to be statistically significant.

\section{RESULTS}

Table 2 lists anthropometric characteristics of the participants included in the analysis. A total of $44.8 \%$ of the participants had normal weight, and $55.2 \%$ were overweight or obese. There were no significant differences in age, height, diastolic blood pressure, or heart rate between groups. Weight, BMI, WC, HC, WHR, and RMR were highest in MUO, followed by MHO, NWO, and MHNW in that order. Total body water was significantly higher in obese groups (MUO, MHO) than in normal weight individuals. Systolic blood pressure was significantly higher in MUO than in the other three groups (Table 2). As shown in Tables 3 and 4, the amount of fat in all parts of the body was higher in NWO than in MHNW, but this difference was not observed for LBMs. Body fat and LBM of all body parts was higher in obese groups (MUO and MHO) than in normal weight groups (NWO and MHNW). Also, body fat and LBM (expect fat free mass, left and right foot) were higher in MUO than MHO (Table 3 and 4). As shown in Tables 5 and 6, there were no significant differences between NHNW and NWO in lipid profile, glycemic status, or liver enzymes. The MUO group had significantly higher values for TG, VLDL, LDL/HDL, Cholesterol/HDL, AIP, FBS, and hsCRP than the other three groups. Furthermore, the lowest level of HDL was observed in the MUO group. LDL/HDL and hs-CRP were higher in MHO participants than in MHNW participants, whereas HDL-c was higher in MHNW than MHO.

Table 2. Anthropometric status by group.

\begin{tabular}{|c|c|c|c|c|c|}
\hline \multirow{2}{*}{ Value } & MHNW & NWO & $\mathrm{MHO}$ & MUO & \multirow{2}{*}{$\mathrm{P}^{*}$} \\
\hline & $(n=53)$ & $(n=29)$ & $(n=57)$ & $(n=44)$ & \\
\hline Age & $26.36 \pm 5.01$ & $27.21 \pm 4.61$ & $28.44 \pm 4.53$ & $27.09 \pm 4.30$ & 0.13 \\
\hline Weight & $56.33 \pm 6.70^{\mathrm{b}, \mathrm{c}}$ & $58.69 \pm 4.58^{\mathrm{d}, e}$ & $83.93 \pm 14.85^{f}$ & $93.12 \pm 14.91$ & $<0.001$ \\
\hline Height & $159.44 \pm 5.42$ & $158.62 \pm 4.93$ & $159.62 \pm 5.31$ & $158.30 \pm 4.83$ & 0.55 \\
\hline LBM & $21.02 \pm 2.29^{\mathrm{b}, \mathrm{c}}$ & $20.42 \pm 2.05^{\mathrm{d}, e}$ & $25.80 \pm 4.74$ & $26.75 \pm 3.50$ & $<0.001$ \\
\hline $\mathrm{FM}$ & $17.39 \pm 4.23^{\mathrm{b}, \mathrm{c}}$ & $20.72 \pm 2.13^{\mathrm{d}, e}$ & $37.77 \pm 10.35^{f}$ & $45.14 \pm 10.13$ & $<0.001$ \\
\hline TBW & $28.52 \pm 2.80^{b, c}$ & $27.77 \pm 2.48^{\mathrm{d}, e}$ & $33.85 \pm 4.51$ & $35.21 \pm 4.26$ & $<0.001$ \\
\hline FFM & $38.90 \pm 3.81^{b, c}$ & $37.91 \pm 3.39^{\mathrm{d}, e}$ & $45.62 \pm 5.37$ & $47.92 \pm 5.77$ & $<0.001$ \\
\hline $\mathrm{BMI}$ & $22.14 \pm 1.85^{b, c}$ & $23.29 \pm 1.21^{\mathrm{d}, e}$ & $33.19 \pm 6.37$ & $37.17 \pm 5.53$ & $<0.001$ \\
\hline BFP & $30.60 \pm 5.66^{a, b, c}$ & $35.32 \pm 2.45^{\mathrm{d}, e}$ & $44.63 \pm 5.00$ & $48.00 \pm 4.21$ & $<0.001$ \\
\hline WHR & $0.76 \pm 0.06^{\mathrm{b}, \mathrm{c}}$ & $0.77 \pm 0.05^{\mathrm{d}, \mathrm{e}}$ & $0.86 \pm 0.07$ & $0.87 \pm 0.05$ & $<0.001$ \\
\hline WC & $71.20 \pm 5.65^{b, c}$ & $74.25 \pm 4.53^{\mathrm{d}, e}$ & $95.82 \pm 12.90^{f}$ & $102.29 \pm 9.79$ & $<0.001$ \\
\hline $\mathrm{HC}$ & $93.04 \pm 5.09^{\mathrm{b}, \mathrm{c}}$ & $95.72 \pm 4.48^{\mathrm{d}, \mathrm{e}}$ & $111.03 \pm 9.11^{f}$ & $116.25 \pm 7.40$ & $<0.001$ \\
\hline $\mathrm{HR}$ & $93.96 \pm 14.07$ & $90.34 \pm 13.42$ & $92.75 \pm 12.75$ & $88.41 \pm 11.23$ & 0.16 \\
\hline SBP & $113.77 \pm 11.09^{c}$ & $115.70 \pm 11.27^{e}$ & $115.37 \pm 14.31^{f}$ & $129.28 \pm 14.18$ & $<0.001$ \\
\hline DBP & $75.94 \pm 9.96$ & $76.93 \pm 10.81$ & $75.03 \pm 15.18^{f}$ & $81.83 \pm 13.74$ & 0.05 \\
\hline $\mathrm{RMR}$ & $1333.01 \pm 81.34^{\mathrm{b}, \mathrm{c}}$ & $1310.25 \pm 81.34^{\mathrm{d}, \mathrm{e}}$ & $1501.83 \pm 126.47$ & $1547.36 \pm 136.94$ & $<0.001$ \\
\hline
\end{tabular}

MHNW = metabolically healthy normal weight; NWO = normal weight obese; $\mathrm{MHO}=$ metabolically healthy obese; $\mathrm{MUO}=$ metabolically unhealthy obese; $\mathrm{LBM}=$ lean body mass; $F M=$ fat mass; $T B W=$ total body water; FFM = fat free mass; $B M I=$ body mass index; $B F P=$ percentage of body fat; WHR = waist-to-hip ratio; $\mathrm{WC}=$ waist circumference; $\mathrm{HC}=$ hip circumference; $\mathrm{HR}=$ heart rate; $\mathrm{SBP}=$ systolic blood pressure; $\mathrm{DBP}=$ diastolic blood pressure; $\mathrm{RMR}=$ resting metabolic rate. "P-value of the comparison between groups. asignificant difference between $\mathrm{MHNW}$ and NWO; 'significant difference between $\mathrm{MHNW}$ and $\mathrm{MHO}$; ${ }^{\mathrm{s}}$ significant difference between MHNW and MUO; ${ }^{d}$ significant difference between NWO and MHO; ${ }^{\mathrm{e}}$ significant difference between NWO and MUO; ${ }^{\mathrm{f}}$ significant difference between $\mathrm{MHO}$ and $\mathrm{MUO}$. 
Table 3. Body composition by group.

\begin{tabular}{|c|c|c|c|c|c|}
\hline \multirow{2}{*}{ Value } & MHNW & NWO & $\mathrm{MHO}$ & MUO & \multirow{2}{*}{$\mathrm{P}^{*}$} \\
\hline & $(n=53)$ & $(n=29)$ & $(n=57)$ & $(n=44)$ & \\
\hline LBM (left arm) & $1.82 \pm 0.27^{\mathrm{a}, \mathrm{b}}$ & $1.78 \pm 0.25^{\mathrm{c,d}}$ & $2.46 \pm 0.42^{\mathrm{e}}$ & $2.66 \pm 0.43$ & $<0.001$ \\
\hline LBM (right arm) & $1.85 \pm 0.28^{\mathrm{a}, \mathrm{b}}$ & $1.81 \pm 0.27^{\mathrm{c}, \mathrm{d}}$ & $2.47 \pm 0.41^{\mathrm{e}}$ & $2.68 \pm 0.42$ & $<0.001$ \\
\hline LBM (trunk) & $17.33 \pm 1.80^{\mathrm{a}, \mathrm{b}}$ & $17.15 \pm 1.74^{\mathrm{c}, \mathrm{d}}$ & $21.14 \pm 2.50^{\mathrm{e}}$ & $22.37 \pm 2.61$ & $<0.001$ \\
\hline LBM (left foot) & $5.89 \pm 0.75^{\mathrm{a}, \mathrm{b}}$ & $5.71 \pm 0.66^{\mathrm{c}, \mathrm{d}}$ & $6.83 \pm 0.86$ & $7.18 \pm 0.99$ & $<0.001$ \\
\hline LBM (right foot) & $5.89 \pm 0.75^{\mathrm{a}, \mathrm{b}}$ & $5.72 \pm 0.67^{\mathrm{c}, \mathrm{d}}$ & $6.89 \pm 0.85$ & $7.20 \pm 1.04$ & $<0.001$ \\
\hline LBM (kg) & $21.02 \pm 2.29^{\mathrm{a}, \mathrm{b}}$ & $20.42 \pm 2.05^{\mathrm{cd} d}$ & $25.80 \pm 4.74$ & $26.75 \pm 3.50$ & $<0.001$ \\
\hline FFM & $38.90 \pm 3.81^{\mathrm{a}, \mathrm{b}}$ & $37.91 \pm 3.39^{c, d}$ & $45.62 \pm 5.37$ & $47.92 \pm 5.77$ & $<0.001$ \\
\hline
\end{tabular}

MHNW = metabolically healthy normal weight; $\mathrm{NWO}=$ normal weight obese; $\mathrm{MHO}=$ metabolically healthy obese; $\mathrm{MUO}=$ metabolically unhealthy obese; $\mathrm{LBM}$ = lean body mass; FFM = fat free mass; BMI = body mass index; BFP = percentage of body fat. "P-value of the comparison between groups. asignificant difference between MHNW and MHO; 'bsignificant difference between MHNW and MUO; 'significant difference between NWO and MHO; 'significant difference between NWO and MUO; esignificant difference between MHO and MUO.

Table 4. Body fat status by group.

\begin{tabular}{|c|c|c|c|c|c|}
\hline \multirow{2}{*}{ Value } & MHNW & NWO & $\mathrm{MHO}$ & MUO & \multirow{2}{*}{$\mathrm{P}^{*}$} \\
\hline & $(n=53)$ & $(n=29)$ & $(n=57)$ & $(n=44)$ & \\
\hline FM (left arm) & $1.20 \pm 0.36^{\mathrm{a}, \mathrm{b}, \mathrm{c}}$ & $1.48 \pm 0.21^{\mathrm{d}, e}$ & $3.55 \pm 1.58^{f}$ & $4.55 \pm 1.58$ & $<0.001$ \\
\hline BFP (left arm) & $37.83 \pm 7.96^{\mathrm{a}, \mathrm{b}, \mathrm{c}}$ & $43.99 \pm 4.64^{\mathrm{d}, e}$ & $55.62 \pm 7.50^{f}$ & $60.32 \pm 7.07$ & $<0.001$ \\
\hline FM (right arm) & $1.17 \pm 0.35^{\mathrm{a}, \mathrm{b}, \mathrm{c}}$ & $1.46 \pm 0.19^{\mathrm{d}, e}$ & $3.51 \pm 1.56^{f}$ & $4.52 \pm 1.60$ & $<0.001$ \\
\hline BFP (right arm) & $36.94 \pm 8.01^{\mathrm{a}, \mathrm{b}, \mathrm{c}}$ & $43.25 \pm 4.62^{\mathrm{d}, e}$ & $55.22 \pm 7.77^{f}$ & $61.60 \pm 3.54$ & $<0.001$ \\
\hline FM (trunk) & $8.56 \pm 2.35^{\mathrm{a}, \mathrm{b}, \mathrm{c}}$ & $10.41 \pm 1.23^{\mathrm{d}, e}$ & $18.53 \pm 4.31^{f}$ & $21.31 \pm 3.80$ & $<0.001$ \\
\hline BFP (trunk) & $31.40 \pm 5.99^{\mathrm{a}, \mathrm{b}, \mathrm{c}}$ & $36.38 \pm 2.41^{\mathrm{d}, e}$ & $44.75 \pm 3.87^{f}$ & $47.12 \pm 3.08$ & $<0.001$ \\
\hline FM (left foot) & $2.69 \pm 0.59^{\mathrm{a}, \mathrm{b}, \mathrm{c}}$ & $3.15 \pm 0.32^{\mathrm{d}, e}$ & $5.49 \pm 1.51^{f}$ & $6.57 \pm 1.71$ & $<0.001$ \\
\hline BFP (left foot) & $30.02 \pm 5.32^{\mathrm{a}, \mathrm{b}, \mathrm{c}}$ & $34.26 \pm 2.88^{\mathrm{d}, e}$ & $42.52 \pm 5.07^{f}$ & $45.80 \pm 4.88$ & $<0.001$ \\
\hline FM (right foot) & $2.70 \pm 0.58^{\mathrm{a}, \mathrm{b}, \mathrm{c}}$ & $3.15 \pm 0.31^{\mathrm{d}, e}$ & $5.53 \pm 1.53^{f}$ & $6.62 \pm 1.75$ & $<0.001$ \\
\hline BFP (right foot) & $30.11 \pm 5.01^{\mathrm{a}, \mathrm{b}, \mathrm{c}}$ & $34.27 \pm 2.83^{\mathrm{d}, e}$ & $42.45 \pm 5.15^{f}$ & $45.88 \pm 4.82$ & $<0.001$ \\
\hline FM/LBM & $0.83 \pm 0.22^{\mathrm{a}, \mathrm{b}, \mathrm{c}}$ & $1.02 \pm 0.11^{\mathrm{d}, e}$ & $1.46 \pm 0.31^{f}$ & $1.69 \pm 0.27$ & $<0.001$ \\
\hline FM/LBM (left arm) & $0.67 \pm 0.23^{\mathrm{a}, \mathrm{b}, \mathrm{c}}$ & $0.84 \pm 0.15^{\mathrm{d}, e}$ & $1.40 \pm 0.44^{f}$ & $1.69 \pm 0.47$ & $<0.001$ \\
\hline FM/LBM (right arm) & $0.65 \pm 0.23^{\mathrm{a}, \mathrm{b}, \mathrm{c}}$ & $0.82 \pm 0.15^{\mathrm{d}, e}$ & $1.38 \pm 0.45^{f}$ & $1.67 \pm 0.48$ & $<0.001$ \\
\hline FM/LBM (trunk) & $0.50 \pm 0.13^{\mathrm{a}, \mathrm{b}, \mathrm{c}}$ & $0.60 \pm 0.06^{\mathrm{d}, e}$ & $0.86 \pm 0.13^{f}$ & $0.95 \pm 0.11$ & $<0.001$ \\
\hline FM/LBM (left foot) & $0.46 \pm 0.12^{\mathrm{a}, \mathrm{b}, \mathrm{c}}$ & $0.55 \pm 0.07^{\mathrm{d}, e}$ & $0.80 \pm 0.17^{f}$ & $0.91 \pm 0.17$ & $<0.001$ \\
\hline FM/LBM (right foot) & $0.46 \pm 0.11^{\mathrm{a}, \mathrm{b}, \mathrm{c}}$ & $0.55 \pm 0.07^{\mathrm{d}, e}$ & $0.79 \pm 0.17^{f}$ & $0.91 \pm 0.17$ & $<0.001$ \\
\hline $\mathrm{FM}(\mathrm{kg})$ & $17.39 \pm 4.23^{\mathrm{b}, \mathrm{c}}$ & $20.72 \pm 2.13^{\mathrm{d}, e}$ & $37.77 \pm 10.35^{f}$ & $45.14 \pm 10.13$ & $<0.001$ \\
\hline $\mathrm{BF}(\%)$ & $30.60 \pm 5.66^{\mathrm{a}, \mathrm{b}, \mathrm{c}}$ & $35.32 \pm 2.45^{\mathrm{d}, e}$ & $44.63 \pm 5.00$ & $48.00 \pm 4.21$ & $<0.001$ \\
\hline
\end{tabular}

MHNW = metabolically healthy normal weight; $\mathrm{NWO}=$ normal weight obese; $M H O=$ metabolically healthy obese; $M U O=$ metabolically unhealthy obese; $\mathrm{LBM}=$ lean body mass; FM = fat mass; BFP = percentage of body fat; BF = body fat. "P-value of the comparison between groups. ${ }^{\mathrm{a}}$ significant difference between MHNW and NWO; ${ }^{b}$ significant difference between MHNW and MHO; ${ }^{c}$ significant difference between MHNW and MUO; ${ }^{d}$ significant difference between NWO and $\mathrm{MHO}$; ${ }^{e}$ significant difference between NWO and MUO; 'significant difference between MHO and MUO

Table 5. Lipid profiles by group.

\begin{tabular}{|c|c|c|c|c|c|}
\hline \multirow{2}{*}{ Value } & MHNW & NWO & $\mathrm{MHO}$ & MUO & \multirow{2}{*}{$\mathrm{P}^{*}$} \\
\hline & $(n=53)$ & $(n=29)$ & $(n=57)$ & $(n=44)$ & \\
\hline Cholesterol & $174.13 \pm 24.79$ & $179.89 \pm 29.15$ & $180.86 \pm 30.31$ & $187.45 \pm 36.18$ & 0.19 \\
\hline Triglycerides & $100.52 \pm 49.93^{b}$ & $102.79 \pm 64.52^{c}$ & $100.94 \pm 33.06^{d}$ & $157.50 \pm 47.96$ & $<0.001$ \\
\hline $\mathrm{HDL}$ & $52.18 \pm 7.56^{\mathrm{a}, \mathrm{b}}$ & $49.10 \pm 7.59^{c}$ & $47.77 \pm 9.13^{d}$ & $37.79 \pm 6.04$ & $<0.001$ \\
\hline LDL & $101.15 \pm 23.66^{b}$ & $107.20 \pm 28.71$ & $113.80 \pm 28.28$ & $120.81 \pm 34.83$ & 0.008 \\
\hline LDL/HDL & $2.01 \pm 0.77^{\mathrm{a}, \mathrm{b}}$ & $2.28 \pm 0.94^{c}$ & $2.55 \pm 0.97^{d}$ & $3.29 \pm 1.10$ & $<0.001$ \\
\hline Cholesterol/HDL & $3.45 \pm 1.04^{b}$ & $3.82 \pm 1.30^{c}$ & $3.99 \pm 1.08^{\mathrm{d}}$ & $5.10 \pm 1.27$ & $<0.001$ \\
\hline VLDL & $20.10 \pm 9.98^{b}$ & $20.55 \pm 12.90^{c}$ & $20.19 \pm 6.61^{d}$ & $31.50 \pm 9.59$ & $<0.001$ \\
\hline AIP & $0.61 \pm 0.19^{\mathrm{b}}$ & $0.63 \pm 0.24^{c}$ & $0.67 \pm 0.17^{d}$ & $0.96 \pm 0.17$ & $<0.001$ \\
\hline
\end{tabular}

MHNW = metabolically healthy normal weight; NWO = normal weight obese; $\mathrm{MHO}=$ metabolically healthy obese; $\mathrm{MUO}=$ metabolically unhealthy obese.; $\mathrm{HDL}=$ High-density lipoprotein; $\mathrm{LDL}=\mathrm{Low}$-density lipoprotein; $\mathrm{VLDL}=$ Very-low-density lipoprotein; AIP = Atherogenic index of plasma. "P-value of the comparison between groups. ${ }^{a}$ significant difference between MHNW and MHO; ${ }^{b}$ significant difference between MHNW and MUO; 'significant difference between NWO and MUO; 'significant difference between $\mathrm{MHO}$ and MUO. 
Table 6. Biochemical factors by group.

\begin{tabular}{|c|c|c|c|c|c|}
\hline \multirow{2}{*}{ Value } & MHNW & NWO & $\mathrm{MHO}$ & MUO & \multirow{2}{*}{$\mathrm{P}^{*}$} \\
\hline & $(n=53)$ & $(n=29)$ & $(n=57)$ & $(n=44)$ & \\
\hline AST & $24.26 \pm 18.46$ & $26.55 \pm 24.10$ & $23.63 \pm 9.22$ & $23.81 \pm 12.29$ & 0.86 \\
\hline ALT & $20.03 \pm 11.06$ & $21.06 \pm 13.94$ & $23.94 \pm 9.93$ & $23.95 \pm 13.67$ & 0.25 \\
\hline ALP & $181.01 \pm 42.82$ & $185.58 \pm 37.39$ & $184.51 \pm 59.87$ & $195.38 \pm 64.40$ & 0.6 \\
\hline FBS & $87.28 \pm 9.27^{b}$ & $89.27 \pm 9.23$ & $88.31 \pm 9.28^{d}$ & $93.68 \pm 11.14$ & 0.01 \\
\hline Insulin & $9.70 \pm 5.07$ & $10.69 \pm 5.96$ & $11.32 \pm 6.52$ & $12.61 \pm 7.96$ & 0.17 \\
\hline HOMA_IR & $1.23 \pm 0.63$ & $1.36 \pm 0.74$ & $1.43 \pm 0.80$ & $1.62 \pm 1.01$ & 0.13 \\
\hline HOMA-s\% & $99.58 \pm 45.20$ & $95.85 \pm 53.09$ & $90.73 \pm 48.47$ & $82.03 \pm 41.41$ & 0.31 \\
\hline HOMA-b\% & $119.23 \pm 48.13$ & $121.72 \pm 56.15$ & $129.34 \pm 58.50$ & $122.13 \pm 49.60$ & 0.78 \\
\hline hsCRP & $1.56 \pm 1.24^{\mathrm{a}, \mathrm{b}}$ & $2.08 \pm 1.10^{c}$ & $4.09 \pm 5.18^{d}$ & $6.64 \pm 5.54$ & $<0.001$ \\
\hline
\end{tabular}

MHNW = metabolically healthy normal weight; NWO = normal weight obese; $M H O=$ metabolically healthy obese; $M U O=$ metabolically unhealthy obese AST = aspartate aminotransferase; $A L T=$ alanine aminotransferase; $A L P=$ alkaline phosphatase; $F B S=$ fasting blood sugar; HOMA_IR = Homeostatic Model Assessment for Insulin Resistance; HOMA-s\% = HOMA of insulin sensitivity; HOMA-b\% = HOMA of $\beta$-cell function; hsCRP = High sensitivity C-reactive protein "P-value of the comparison between groups. ${ }^{a}$ significant difference between MHNW and MHO; ${ }^{b}$ significant difference between MHNW and MUO; ${ }^{c}$ significant difference between NWO and MUO; 'significant difference between $\mathrm{MHO}$ and $\mathrm{MUO}$.

\section{DISCUSSION}

Our analysis revealed that MHO individuals were at increased risk of CVD incidents, compared with normal weight individuals without metabolic syndrome. However, MHO individuals display a partially more favorable metabolic profile than MUO individuals. Despite the increasing number of studies evaluating $\mathrm{MHO}$, investigations of the association between metabolically healthy obesity and CVD risk have reported controversial results. Several studies have observed no elevated risk of CVD in MHO individuals, whereas some other studies have shown an elevated risk of CVD in this phenotype. ${ }^{13-15}$ In one study of women aged 45 years and over, with 10 years of follow-up, no increased CVD risk for MHO phenotypes was observed. ${ }^{13}$ In another study, in adults aged 35-55 years, it was shown that MHO individuals were at increased risk of CVD incidents, compared with MHNW individuals. ${ }^{14}$ Karelis et al. ${ }^{15}$ indicated that postmenopausal women displaying the MHO phenotype also have a favorable inflammation profile, as shown by lower CRP and $\alpha-1$ antitrypsin levels compared with insulin-resistant women. These authors suggested that a satisfactory inflammation profile, as verified by low CRP levels, could play a role in the protective profile of the MHO individual and that this may be related metabolically to a lower risk of cardiovascular disease..$^{15}$ Also, we found that a low inflammation state was a part of the protective profile of the MHO subgroup, when compared to the MUO subgroup. We observed a lower hs-CRP concentration in MHO participants than MUO participants ( $4.09 \pm 5.18$ vs $6.64 \pm 5.54$, respectively). However, the MHO subgroup's mean hs-CRP level was higher than the JUPITER threshold, $\geq 2 \mathrm{mg} / \mathrm{L}$ and also higher than that of the MHNW subgroup. ${ }^{16}$
The second favorable aspect of the MHO metabolic profile compared to the MUO profile was a higher HDL level, and lower levels of fasting TG, LDL/HDL, and Cholesterol/HDL. An adequate lipid profile has been reported as a protective factor for cardiovascular disease. ${ }^{17}$ Another variable associated with a more favorable metabolic profile in $\mathrm{MHO}$ in compared to MUO was FBS. We also found that young MHO women had no increased risk of developing type 2 diabetes compared with MHNW. Finally, one other protective variable was lower AIP in MHO participants compared with MUO subjects. This variable was not statistically different between $\mathrm{MHO}$ and $\mathrm{MHNW}$ or NWO participants. In a study by Koborová et al. ${ }^{18}$ AIP was significantly lower in metabolically healthy obese women than in unhealthy centrally obese women, which is similar to our findings. Also, a study by Chhezom showed that obese individuals had a significantly higher atherogenic index of plasma compared to normal weight and overweight individuals. ${ }^{19}$ Recently, AIP was identified as a useful and novel marker for the risk of atherosclerosis and cardiovascular disease. ${ }^{20}$ This marker reflects the delicate metabolic interactions within the whole lipoprotein complex and is a better predictor of atherogenic risk than triglyceride and HDL-C levels evaluated separately. ${ }^{21}$

It is likely that $\mathrm{MHO}$ is a transient condition..$^{5,22}$ Thus, in MHO individuals, sustaining factors such as a healthy diet, smoking cessation, preservation of metabolic health, and physical activity may prevent progression to the MUO phenotype..$^{4-7}$ Dhana et al. ${ }^{23}$ suggested that the metabolic status of MHO individuals should be reassessed on a regular basis.

We employed a cross-sectional approach, so we couldn't establish relationships of causality. Despite this limitation, our results are strengthened by measurement of body composition, liver function 
enzymes, complete lipid profile, glycemic indexes and inflammatory markers in young Iranian women.

In conclusion, results of the present study demonstrate that young women displaying the MHO phenotype have a favorable metabolic profile as shown by lower FBS, TG, LDL-c/HDL-c, Cho/HDL-c, hs-CRP, and AIP, and higher HDL-c levels than those with the MUO phenotype. However, MHO individuals were nevertheless at increased risk of CVD incident (lower HDL-C and more hs-CRP level), compared with MHNW individuals. As mentioned above, the inconsistent definition of MHO among studies is the main obstacle to advancing our understanding of the MHO phenotype. It is therefore necessary to standardize the definition of MHO.

\section{REFERENCES}

1. Kolahi AA, Moghisi A, Soleiman Ekhtiari Y. Socio-demographic determinants of obesity indexes in Iran: findings from a nationwide STEPS survey. Health Promot Perspect. 2018;8(3):187-94. http:// dx.doi.org/10.15171/hpp.2018.25. PMid:30087841.

2. Tabrizi JS, Sadeghi-Bazargani H, Farahbakhsh M, Nikniaz L, Nikniaz Z. Prevalence and associated factors of overweight or obesity and abdominal obesity in iranian population: a population-based study of Northwestern Iran. Iran J Public Health. 2018;47(10):1583-92. PMid:30524990.

3. Ruderman NB, Schneider SH, Berchtold P. The "metabolically-obese," normal-weight individual. Am J Clin Nutr. 1981;34(8):1617-21. http://dx.doi.org/10.1093/ajcn/34.8.1617. PMid:7270486.

4. Seo $\mathrm{MH}$, Rhee EJ. Metabolic and cardiovascular implications of a metabolically healthy obesity phenotype. Endocrinol Metab. 2014;29(4):427-34. http://dx.doi.org/10.3803/EnM.2014.29.4.427. PMid:25559571.

5. Jung $\mathrm{CH}$, Lee $\mathrm{WJ}$, Song $\mathrm{KH}$. Metabolically healthy obesity: a friend or foe? Korean J Intern Med. 2017;32(4):611-21. http://dx.doi. org/10.3904/kjim.2016.259. PMid:28602062.

6. Stefan N, Haring HU, Hu FB, Schulze MB. Metabolically healthy obesity: epidemiology, mechanisms, and clinical implications. Lancet Diabetes Endocrinol. 2013;1(2):152-62. http://dx.doi. org/10.1016/S2213-8587(13)70062-7. PMid:24622321.

7. Samocha-Bonet D, Dixit VD, Kahn CR, et al. Metabolically healthy and unhealthy obese: the 2013 Stock Conference report. Obes Rev. 2014;15(9):697-708. http://dx.doi.org/10.1111/obr.12199. PMid:25059108.

8. Blüher $M$. Are metabolically healthy obese individuals really healthy? Eur J Endocrinol. 2014;171(6):R209-19. http://dx.doi. org/10.1530/EJE-14-0540. PMid:25012199.

9. Phillips CM. Metabolically healthy obesity: definitions, determinants and clinical implications. Rev Endocr Metab Disord. 2013;14(3):21927. http://dx.doi.org/10.1007/s11154-013-9252-x. PMid:23928851.

10. Huang PL. A comprehensive definition for metabolic syndrome. Dis Model Mech. 2009;2(5-6):231-7. http://dx.doi.org/10.1242/ dmm.001180. PMid:19407331.
11. Ashtary-Larky D, Daneghian S, Alipour M, et al. Waist circumference to height ratio: better correlation with fat mass than other anthropometric indices during dietary weight loss in different rates. Int J Endocrinol Metab. 2018;16(4):e55023. http://dx.doi. org/10.5812/ijem.55023. PMid:30464770.

12. Wallace TM, Levy JC, Matthews DR. Use and abuse of HOMA modeling. Diabetes Care. 2004;27(6):1487-95. http://dx.doi. org/10.2337/diacare.27.6.1487. PMid:15161807.

13. Song Y, Manson JE, Meigs JB, Ridker PM, Buring JE, Liu S. Comparison of usefulness of body mass index versus metabolic risk factors in predicting 10-year risk of cardiovascular events in women. Am J Cardiol. 2007;100(11):1654-8. http://dx.doi.org/10.1016/j. amjcard.2007.06.073. PMid:18036364.

14. Hinnouho GM, Czernichow S, Dugravot A, et al. Metabolically healthy obesity and the risk of cardiovascular disease and type 2 diabetes: the Whitehall II cohort study. Eur Heart J. 2015;36(9):5519. PMid:24670711.

15. Karelis AD, Faraj M, Bastard J-P, et al. The Metabolically Healthy but Obese Individual Presents a Favorable Inflammation Profile. J Clin Endocrinol Metab. 2005;90(7):4145-50. http://dx.doi.org/10.1210/ jc.2005-0482. PMid:15855252.

16. Blaha MJ, Rivera JJ, Budoff MJ, et al. Association between obesity, high-sensitivity C-reactive protein $\geq 2 \mathrm{mg} / \mathrm{L}$, and subclinical atherosclerosis: implications of JUPITER from the Multi-Ethnic Study of Atherosclerosis. Arterioscler Thromb Vasc Biol. 2011;31(6):1430-8. http://dx.doi.org/10.1161/ATVBAHA.111.223768. PMid:21474823.

17. Kutkiene S, Petrulioniene Z, Laucevicius A, et al. Cardiovascular risk profile of patients with atherogenic dyslipidemia in middle age Lithuanian population. Lipids Health Dis. 2018;17(1):208. http://dx.doi.org/10.1186/s12944-018-0851-0. PMid:30185180.

18. Koborová I, Gurecká R, Csongová $M$, et al. Association between metabolically healthy central obesity in women and levels of soluble receptor for advanced glycation end products, soluble vascular adhesion protein-1, and the activity of semicarbazidesensitive amine oxidase. Croat Med J. 2017;58(2):106-16. http:// dx.doi.org/10.3325/cmj.2017.58.106. PMid:28409494.

19. Chhezom K, Arslan MI, Hoque MM, Biswas SK. Biomarkers of cardiovascular and metabolic diseases in otherwise healthy overweight subjects in Bangladesh. Diabetes Metab Syndr. 2017;11(Suppl Suppl 1):S381-4. http://dx.doi.org/10.1016/j. dsx.2017.03.021. PMid:28285989.

20. Cure E, Icli A, Uslu AU, et al. Atherogenic index of plasma: a useful marker for subclinical atherosclerosis in ankylosing spondylitis. Clin Rheumatol. 2018;37(5):1273-80. http://dx.doi.org/10.1007/ s10067-018-4027-0. PMid:29435680.

21. Dobiás囚ová M, Frohlich J. The plasma parameter log (TG/HDL-C) as an atherogenic index: correlation with lipoprotein particle size and esterification rate inapob-lipoprotein-depleted plasma (FERHDL). Clin Biochem. 2001;34(7):583-8. http://dx.doi.org/10.1016/S00099120(01)00263-6. PMid:11738396

22. Lee SK, Kim SH, Cho GY, et al. Obesity phenotype and incident hypertension: a prospective community-based cohort study. J Hypertens. 2013;31(1):145-51. http://dx.doi.org/10.1097/ HJH.0b013e32835a3637. PMid:23079679.

23. Dhana K, Koolhaas CM, Van Rossum EF, et al. Metabolically healthy obesity and the risk of cardiovascular disease in the elderly population. PLoS One. 2016;11(4):e0154273. http://dx.doi. org/10.1371/journal.pone.0154273. PMid:27100779. 
Correspondence Meysam Alipour Nutrition and Metabolic Diseases Research Center, Ahvaz Jundishapur University of Medical Sciences P. O. Box 159613-5715794 - Ahvaz, Iran Tel.: +986133367543

E-mail: meysam.aalipour@yahoo.com

Author information SAH - PhD in Biochemistry, Ahvaz Jundishapur University of Medical Sciences.

VA - PhD in Nutrition, Khalkhal University of Medical Sciences. DAL and NLD - MSc in Biochemistry, Ahvaz Jundishapur University of Medical Sciences.

MA and MG - PhD in Nutrition, Ahvaz Jundishapur University of Medical Sciences.

Author contributions Conception and design: SAH Analysis and interpretation: VA

Data collection: NLD Writing the article: MA Critical revision of the article: DAL Final approval of the article*: SAH, VA, DAL, MA, MG, NLD Statistical analysis: MG Overall responsibility: SAH

*All authors have read and approved of the final version of the article submitted to I Vasc Bras. 